\title{
Baseline hematology and serum biochemistry results for Indian leopards (Panthera pardus fusca)
}

\author{
Arun Attur Shanmugam ${ }^{1,2}$, Sanath Krishna Muliya ${ }^{3}$, Ajay Deshmukh ${ }^{4}$, Sujay Suresh ${ }^{3}$, Anukul Nath ${ }^{5}$, Pa Kalaignan $^{3}$, \\ Manjunath Venkataravanappa ${ }^{6}$ and Lyju Jose ${ }^{1}$
}

1. Department of Biotechnology, Jain University, Bengaluru, Karnataka, India; 2. Wildlife SOS, Bengaluru, Karnataka, India; 3. Bannerghatta Biological Park, Bannerghatta, Bengaluru, Karnataka, India; 4. Manikdoh Leopard Rescue Center, Wildlife SOS, Khamgaon, Maharashtra, India; 5. Department of Ecology \& Environmental Science, E. P. Odum School of Environmental Sciences, Silchar, Assam, India; 6. Wild Animal Disease Diagnostic Lab, Bannerghatta Biological Park, Bannerghatta, Bengaluru, Karnataka, India.

Corresponding author: Sanath Krishna Muliya, e-mail: sanamuliya@yahoo.co.in

Co-authors: AAS: arun@wildlifesos.org, AD: ajay@wildlifesos.org, SS: newtownsujay@yahoo.co.in,

AN: anukul@wii.gov.in, PK: drkalai.wildvet@gmail.com, MV: manjuvet330@gmail.com, LJ: lyjujose@gmail.com

Received: 09-02-2017, Accepted: 01-06-2017, Published online: 24-07-2017

doi: 10.14202/vetworld.2017.818-824 How to cite this article: Shanmugam AA, Muliya SK, Deshmukh A, Suresh S, Nath A, Kalaignan P, Venkatramappa M, Jose L (2017) Baseline hematology and serum biochemistry results for Indian leopards (Panthera pardus fusca), Veterinary World, 10(7): 818-824.

\begin{abstract}
Aim: The aim of the study was to establish the baseline hematology and serum biochemistry values for Indian leopards (Panthera pardus fusca), and to assess the possible variations in these parameters based on age and gender.

Materials and Methods: Hemato-biochemical test reports from a total of 83 healthy leopards, carried out as part of routine health evaluation in Bannerghatta Biological Park and Manikdoh Leopard Rescue Center, were used to establish baseline hematology and serum biochemistry parameters for the subspecies. The hematological parameters considered for the analysis included hemoglobin (Hb), packed cell volume, total erythrocyte count (TEC), total leukocyte count (TLC), mean corpuscular volume $(\mathrm{MCV})$, mean corpuscular $\mathrm{Hb}(\mathrm{MCH})$, and $\mathrm{MCH}$ concentration. The serum biochemistry parameters considered included total protein (TP), albumin, globulin, aspartate aminotransferase, alanine aminotransferase (ALT), blood urea nitrogen, creatinine, triglycerides, calcium, and phosphorus.

Results: Even though few differences were observed in hematologic and biochemistry values between male and female Indian leopards, the differences were statistically not significant. Effects of age, however, were evident in relation to many hematologic and biochemical parameters. Sub-adults had significantly greater values for Hb, TEC, and TLC compared to adults and geriatric group, whereas they had significantly lower MCV and MCH compared to adults and geriatric group. Among, serum biochemistry parameters the sub-adult age group was observed to have significantly lower values for TP and ALT than adult and geriatric leopards.

Conclusion: The study provides a comprehensive analysis of hematologic and biochemical parameters for Indian leopards. Baselines established here will permit better captive management of the subspecies, serve as a guide to assess the health and physiological status of the free ranging leopards, and may contribute valuable information for making effective management decisions during translocation or rehabilitation process.
\end{abstract}

Keywords: hematology, Indian leopard, Panthera pardus fusca, serum biochemistry.

\section{Introduction}

Leopards are one of the widely distributed wild felids in the world, with nine subspecies found in varied habitats across their extent range [1]. The species is currently categorized as "vulnerable" on the IUCN Red List, with some subspecies identified as "endangered" or "critically endangered" [2]. India hosts substantial numbers of the subspecies Panthera pardus fusca, the Indian leopard which survives in protected areas and multiple use forests all across India, except for the arid deserts and regions above the timberline

Copyright: Shanmugam, et al. Open Access. This article is distributed under the terms of the Creative Commons Attribution 4.0 International License (http://creativecommons.org/licenses/ by/4.0/), which permits unrestricted use, distribution, and reproduction in any medium, provided you give appropriate credit to the original author(s) and the source, provide a link to the Creative Commons license, and indicate if changes were made. The Creative Commons Public Domain Dedication waiver (http:// creativecommons.org/publicdomain/zero/1.0/) applies to the data made available in this article, unless otherwise stated. in the Himalayas [3]. The subspecies is even known to persist close to human populations by feeding on livestock and domestic dogs [4]. Ensuing this, incidences of human-leopard conflicts have also seen a sharp rise in recent past in India, leading to casualties on both sides. This has, in turn, led to increased veterinary interventions in the species. Further, a large population of Indian leopards exit in captivity maintained in almost all the Indian zoos [5].

Given the difficulty in detecting signs of disease and distress in wild animals, comprehensive health assessments, including hematology and serum biochemistry studies, have been crucial to assess the effects of many health-related problems [6]. Such information has also been proven useful to assess the subclinical effects of pathogens [7] and physiological [8,9], ecological [10], or nutritional [7] status, among other issues.

To interpret a laboratory report to be normal or abnormal, the values expected to be obtained from 
healthy animals (reference intervals) must be known first [11]. However, unlike other large felids [12-15], a reliable baseline hematological and serum biochemistry references, that can be used to assess the health status of Indian leopards is still lacking. The reference intervals available are usually based on limited population testing and do not account for variation within the subspecies or within subpopulations defined by age, gender, and other factors [16,17]. Further, these parameters can also be influenced by several additional extrinsic factors such as the presence of pathological condition, phlebotomy techniques used, and chemical immobilization before sampling [18]. Thus, this study was undertaken to establish the baseline reference interval of 7 hematology and 11 serum biochemistry variables from Indian leopards and to assess the influence of age and gender on these variables, while keeping the extrinsic factors constant.

\section{Materials and Methods}

\section{Ethical approval}

Not applicable: None of the animals were sampled solely for the purpose of this study. The study was carried out by compiling and analyzing available hemato-biochemical test reports, carried out as part of routine health evaluations in both the study sites.

\section{Study area and animals}

Hemato-biochemical test reports from a total of 83 healthy leopards, carried out as part of routine health evaluation in Bannerghatta Biological Park, Karnataka (BBP, $\mathrm{n}=41$ ) and Manikdoh Leopard Rescue Center, Maharashtra (MLRC, $n=42$ ) between January 2014 and January 2016 were used to establish baseline hematology and serum biochemistry parameters for the subspecies. The leopards from BBP included both captive born leopards from zoo section and rescued free ranging leopards from rescue center, whereas leopards from MLRC were mainly rescued free ranging leopards. While the reports from animals which appeared to be healthy and clinically stable during sampling were considered, reports from the ones with visible injuries and known history of illness were excluded from the analysis.

\section{Blood collection and processing}

Since it is crucial that samples are obtained under consistent conditions to arrive at baseline value, the restraint and phlebotomy techniques were also considered. Based on available information, uniform protocols were followed in both the institutions. All the leopards considered for this study were physically restrained by securing them in squeeze cages, and phlebotomy was done from lateral coccygeal vein, without any chemical immobilization. Hematological parameters were evaluated using commercially available automated analyzers (BBP: HumaCount 30TS, HUMAN Gesellschaft für Biochemica und Diagnostica $\mathrm{mbH}$, Wiesbaden, Germany; MLRC: BC-1800, Vector Biotek Pvt. Ltd. Gujarat, India), whereas the serum biochemistry assays were performed on semi-automated clinical chemistry analyzers (BBP: ERBA Chem Pro, Transasia House, Mumbai, India; MLRC: VChem+, Vector Biotek Pvt. Ltd. Gujarat, India) using commercially available biochemical kits, calibrated with control reagents before sample analysis. Further, all the samples were confirmed to have undergone laboratory analyses within 2-3 h of collection.

\section{Hemato-biochemical parameters}

The hematological parameters considered for the analysis included hemoglobin ( $\mathrm{Hb}$ in $\mathrm{g} / \mathrm{dl}$ ), packed cell volume (in \%), total erythrocyte count (TEC in $10^{6} / \mu 1$ ), total leukocyte count (TLC in $10^{3} / \mu \mathrm{l}$ ), mean corpuscular volume (MCV in $\mathrm{fl})$, mean corpuscular $\mathrm{Hb}(\mathrm{MCH}$ in $\mathrm{pg}$ ), and $\mathrm{MCH}$ concentration (in $\mathrm{g} / \mathrm{dL}$ ). The serum biochemistry parameters considered included total protein ( $\mathrm{TP}$ in $\mathrm{g} / \mathrm{dl}$ ), albumin (in $\mathrm{g} / \mathrm{dl}$ ), globulin (in $\mathrm{g} / \mathrm{dl}$ ), aspartate aminotransferase (AST in IU/L), alanine aminotransferase (ALT in IU/L), blood urea nitrogen (in $\mathrm{mg} / \mathrm{dl}$ ), creatinine (in $\mathrm{mg} / \mathrm{dl}$ ), triglycerides (in $\mathrm{mg} / \mathrm{dl}$ ), calcium (in $\mathrm{mg} / \mathrm{dl}$ ), and phosphorus (in $\mathrm{mg} / \mathrm{dl}$ ).

\section{Statistical analysis}

For the purpose of statistical analysis, leopards were grouped according to their gender (male, $n=34$ and female, $n=49$ ) and further into three age groups: Sub-adults ( $1-3$ years old; $n=19)$, adults (3-10 years old, $n=38)$ and geriatric animals (10 years and above, $\mathrm{n}=26$ ), based on available zoo/rescue center records. The mean and standard deviation for each hemato-biochemical values obtained were calculated as per the standard procedure [19]. If any value was less than or equal to the first quartile -3 times the interquartile range or greater than the third quartile +3 times the interquartile range, they were regarded as extreme outliers and were removed before further analyses and then the middle $95 \%$ of test results were considered as the reference interval. Data were further tested for normality using the Shapiro-Wilk test for normality using R version 3.2.5. The dataset which followed normal distribution was tested using independent t-test to compare the mean of the hematological parameters, whereas the parameters which did not meet the criteria of normality were tested using Mann-Whitney U-tests (two factors) to evaluate significant differences. The variation between different age classes irrespective of gender was tested using one-way ANOVA followed by Tukey test for the data following the normal distribution pattern and KruskalWallis test for the data which did not meet the criteria. Significance level was $\mathrm{p}<0.05$ for all tests.

\section{Results}

The results and descriptive statistics for hemato-biochemistry parameters analyzed in this study are listed in Tables-1-4. Even though few differences were observed in hematologic and biochemistry values between male and female Indian leopards, the differences were statistically not significant. 
Table-1: Overall hematology and serum biochemistry results for Indian leopards (Panthera pardus fusca) from BBP and MLRC.

\begin{tabular}{|c|c|c|c|c|c|c|c|}
\hline \multirow[t]{2}{*}{ Parameters } & \multirow[t]{2}{*}{$\mathbf{n}$} & \multirow[t]{2}{*}{ Minimum } & \multirow[t]{2}{*}{ Maximum } & \multirow[t]{2}{*}{ Mean \pm SD } & \multirow[t]{2}{*}{ SEM } & \multicolumn{2}{|c|}{$\begin{array}{c}95 \% \text { CI of the } \\
\text { mean }\end{array}$} \\
\hline & & & & & & LCI & UCI \\
\hline TLC $\left(10^{3} / \mu \mathrm{l}\right)$ & 83 & 8.0 & 36.9 & $16.2 \pm 6.1$ & 0.7 & 14.9 & 17.5 \\
\hline TEC $\left(10^{6} / \mu \mathrm{l}\right)$ & 83 & 5.4 & 12.7 & $8.7 \pm 2.0$ & 0.2 & 8.3 & 9.1 \\
\hline $\mathrm{Hb}(\mathrm{g} / \mathrm{dl})$ & 83 & 10.5 & 20.2 & $14.7 \pm 2.5$ & 0.3 & 14.2 & 15.3 \\
\hline PCV $(\%)$ & 83 & 32.8 & 65.4 & $48.5 \pm 6.8$ & 0.8 & 47.0 & 50.0 \\
\hline MCV (fl) & 83 & 45.9 & 93.9 & $58.6 \pm 11.3$ & 1.2 & 56.2 & 61.1 \\
\hline $\mathrm{MCH}(\mathrm{pg})$ & 83 & 13.6 & 28.0 & $18.1 \pm 3.0$ & 0.3 & 17.4 & 18.7 \\
\hline $\mathrm{MCHC}(\%)$ & 83 & 21.7 & 36.0 & $30.7 \pm 2.8$ & 0.3 & 30.1 & 31.3 \\
\hline TP $(\mathrm{g} / \mathrm{dl})$ & 83 & 4.3 & 11.1 & $7.0 \pm 1.3$ & 0.1 & 6.7 & 7.3 \\
\hline Albumin (g/dl) & 83 & 1.6 & 7.0 & $3.9 \pm 1.1$ & 0.1 & 3.7 & 4.1 \\
\hline Globulin ( $\mathrm{g} / \mathrm{dl})$ & 83 & 0.3 & 6.6 & $3.0 \pm 1.2$ & 0.1 & 2.8 & 3.3 \\
\hline AST (IU/L) & 41 & 9.9 & 90.8 & $41.3 \pm 19.2$ & 3.0 & 35.5 & 47.2 \\
\hline ALT (IU/L) & 83 & 2.0 & 146.7 & $44.0 \pm 28.2$ & 3.1 & 37.9 & 50.0 \\
\hline CRT (mg/dl) & 83 & 0.4 & 4.2 & $1.6 \pm 0.7$ & 0.1 & 1.4 & 1.7 \\
\hline BUN (mg/dl) & 83 & 3.1 & 56.3 & $27.6 \pm 11.4$ & 1.3 & 25.1 & 30.0 \\
\hline Glucose (mg/dl) & 41 & 14.1 & 159.0 & $58.6 \pm 26.9$ & 4.2 & 50.4 & 66.9 \\
\hline Triglyceride (mg/dl) & 41 & 4.0 & 59.9 & $29.5 \pm 12.5$ & 2.0 & 25.6 & 33.3 \\
\hline $\mathrm{Ca}(\mathrm{mg} / \mathrm{dl})$ & 83 & 1.5 & 27.0 & $9.9 \pm 3.2$ & 0.3 & 9.2 & 10.6 \\
\hline$P(\mathrm{mg} / \mathrm{dl})$ & 83 & 2.2 & 12.4 & $5.5 \pm 1.8$ & 0.2 & 5.1 & 5.9 \\
\hline
\end{tabular}

$\mathrm{TLC}=$ Total leukocyte count, $\mathrm{TEC}=$ Total erythrocyte count, $\mathrm{Hb}=$ Hemoglobin, $\mathrm{PCV}=$ Packed cell volume, $\mathrm{MCV}=\mathrm{Mean}$ corpuscular volume, $\mathrm{MCH}=$ Mean corpuscular hemoglobin, $\mathrm{MCHC}=$ Mean corpuscular hemoglobin concentration, $\mathrm{TP}=\mathrm{Total}$ protein, $\mathrm{AST}=$ Aspartate aminotransferase, $\mathrm{ALT}=\mathrm{Alanine}$ aminotransferase, $\mathrm{BUN}=\mathrm{Blood}$ urea nitrogen, $\mathrm{Ca}=\mathrm{Serum}$ calcium, $\mathrm{P}=$ Serum phosphorus, $\mathrm{CI}=$ Confidence interval, $\mathrm{LCI}=$ Lower confidence interval, UCI=Upper confidence interval, $\mathrm{BBP}=$ Bannerghatta Biological Park, MLRC $=$ Manikdoh Leopard Rescue Center, $\mathrm{SEM}=$ Standard error of mean, $\mathrm{SD}=\mathrm{Standard}$ deviation

Table-2: Comparison of hematology and serum biochemistry results for male and female Indian leopards (Panthera pardus fusca) from BBP and MLRC.

\begin{tabular}{|c|c|c|c|c|c|c|c|c|c|c|c|c|c|c|c|}
\hline \multirow[t]{3}{*}{ Parameters } & \multicolumn{7}{|c|}{ Male } & \multicolumn{7}{|c|}{ Female } & \multirow[t]{3}{*}{ p value } \\
\hline & \multirow[t]{2}{*}{$\mathbf{n}$} & \multirow[t]{2}{*}{ Min } & \multirow[t]{2}{*}{ Max } & \multirow[t]{2}{*}{ Mean \pm SD } & \multirow[t]{2}{*}{ SEM } & \multicolumn{2}{|c|}{$\begin{array}{l}95 \% \text { CI } \\
\text { of the } \\
\text { mean }\end{array}$} & \multirow[t]{2}{*}{$\mathbf{n}$} & \multirow[t]{2}{*}{ Min } & \multirow[t]{2}{*}{ Max } & \multirow[t]{2}{*}{ Mean \pm SD } & \multirow[t]{2}{*}{ SEM } & \multicolumn{2}{|c|}{$\begin{array}{l}95 \% \text { CI } \\
\text { of the } \\
\text { mean }\end{array}$} & \\
\hline & & & & & & LCI & UCI & & & & & & LCI & UCI & \\
\hline $\operatorname{TLC}\left(10^{3} / \mu \mathrm{l}\right)$ & 34 & 8.7 & 36.9 & $17.2 \pm 6.5$ & 1.1 & 15.0 & 19.4 & 49 & 8.0 & 28.2 & $15.5 \pm 5.9$ & 0.8 & 13.9 & 17.0 & 0.37 \\
\hline $\operatorname{TEC}\left(10^{6} / \mu \mathrm{l}\right)$ & 34 & 5.8 & 12.7 & $8.9 \pm 2.2$ & 0.4 & 8.2 & 9.7 & 49 & 5.4 & 11.8 & & 0.3 & 8.0 & 9.1 & .99 \\
\hline $\mathrm{Hb}(\mathrm{g} / \mathrm{dl})$ & 34 & 10.5 & 20.2 & $15.0 \pm$ & 0.5 & 14.1 & 15.9 & 49 & 11.0 & 19.9 & 14. & 0.3 & 14.0 & 15.1 & .57 \\
\hline PCV $(\%)$ & 34 & 32.8 & 65.4 & $49.2 \pm 7.2$ & 1.2 & 46.8 & 51.6 & 49 & 34.0 & 60.7 & 48.0 & 0.9 & 46.2 & 49.7 & .83 \\
\hline $\operatorname{MCV}(\mathrm{fl})$ & 34 & 47.0 & 88.1 & $57.5 \pm 11.1$ & 1.9 & 53.8 & 61.2 & 49 & 45.9 & 93.9 & $59.4 \pm 11.6$ & 1.7 & 56.0 & 62.7 & .45 \\
\hline $\mathrm{MCH}(\mathrm{pg})$ & 34 & 14.2 & 28.0 & $18.3 \pm$ & 0.6 & 17.2 & 19.4 & 49 & 13.6 & 25.0 & 17.9 & 0.4 & 17.1 & 18.6 & 0.59 \\
\hline $\mathrm{CHC}(\%)$ & 34 & 24.4 & 35.1 & $31.1 \pm 2.3$ & 0.4 & 30.3 & 31.9 & 49 & 21.7 & 36.0 & $30.4 \pm 3.0$ & 0.4 & 29.6 & 31.1 & 0.46 \\
\hline$P(g / d l)$ & 34 & 4.6 & 10.4 & $6.8 \pm 1.2$ & 0.2 & 6.4 & 7.2 & 49 & 4.3 & 11.1 & $7.1 \pm 1.4$ & 0.2 & 6.7 & 7.4 & 0.31 \\
\hline Ibumin (g/dl) & 34 & 1.9 & 7.0 & $4.0 \pm 1.1$ & 0.2 & 3.6 & 4.4 & 49 & 1.6 & 6.6 & $3.9 \pm 1.1$ & 0.2 & 3.5 & 4.2 & 0.46 \\
\hline Iobulin (g/dl) & 34 & 0.3 & 5.6 & $2.9 \pm 1.2$ & 0.2 & 2.4 & 3.3 & 49 & 0.3 & 6.6 & $3.2 \pm 1.3$ & 0.2 & 2.8 & 3.5 & 0.66 \\
\hline ST (IU/L) & 22 & 10.6 & 90.8 & $44.7 \pm 21.0$ & 4.5 & 35.9 & 53.4 & 19 & 9.9 & 77.5 & $37.5 \pm 16.8$ & 3.9 & 29.8 & 45.1 & 0.24 \\
\hline LT (IU/L) & 34 & 2.0 & 120.2 & $37.0 \pm 22.9$ & 3.9 & 29.3 & 44.7 & 49 & 3.7 & 146.7 & $48.8 \pm 30.6$ & 4.4 & 40.1 & 57.4 & 0.91 \\
\hline reatinine $(\mathrm{mg} / \mathrm{dl})$ & 34 & 0.5 & 3.0 & $1.5 \pm 0.6$ & 0.1 & 1.3 & 1.7 & 49 & 0.4 & 4.2 & $1.6 \pm 0.7$ & 0.1 & 1.4 & 1.7 & 0.32 \\
\hline BUN ( & 34 & 3.9 & 56.3 & $27.5 \pm 13.6$ & 2.3 & 22.9 & 32.1 & 49 & 3.1 & 49.9 & $27.6 \pm 9.7$ & 1.4 & 24.8 & 30.3 & 0.63 \\
\hline Gluce & 22 & 14.1 & 159.0 & $59.4 \pm 30.7$ & 6.6 & 46.6 & 72.2 & 19 & 14.1 & 95.1 & $57.7 \pm 22.4$ & 5.1 & 47.7 & 67.6 & 0.81 \\
\hline rides $(\mathrm{mg} / \mathrm{dl})$ & 22 & 4.7 & 59.9 & $31.1 \pm 12.0$ & 2.6 & 26.1 & 36.1 & 19 & 4.0 & 52.9 & $27.6 \pm 13.2$ & 3.0 & 21.7 & 33.4 & 0.39 \\
\hline & 34 & 3.5 & 14.7 & $9.6 \pm 2.7$ & 0.5 & 8.7 & 10.5 & 49 & 1.5 & 27.0 & $10.1 \pm 3.5$ & 0.5 & 9.1 & 11.0 & 0.67 \\
\hline$P(\mathrm{mg} / \mathrm{dl})$ & 34 & 2.4 & 12.4 & $5.6 \pm 2.3$ & 0.4 & 4.8 & 6.3 & 49 & 2.2 & 8.9 & $5.5 \pm 1.5$ & 0.2 & 5.1 & 5.8 & 0.70 \\
\hline
\end{tabular}

TLC $=$ Total leukocyte count, TEC $=$ Total erythrocyte count, $\mathrm{Hb}=$ Hemoglobin, $\mathrm{PCV}=$ Packed cell volume, $\mathrm{MCV}=\mathrm{Mean}$ corpuscular volume, $\mathrm{MCH}=$ Mean corpuscular hemoglobin, $\mathrm{MCHC}=$ Mean corpuscular hemoglobin concentration, $\mathrm{TP}=\mathrm{Total}$ protein, AST=Aspartate aminotransferase, ALT=Alanine aminotransferase, BUN=Blood urea nitrogen, Ca=Serum calcium, $\mathrm{P}=$ Serum phosphorus, $\mathrm{CI}=$ Confidence interval, $\mathrm{LCI}=$ Lower confidence interval, UCI=Upper confidence interval, $\mathrm{BBP}=$ Bannerghatta Biological Park, MLRC=Manikdoh Leopard Rescue Center, SEM=Standard error of mean, SD=Standard deviation

Effects of age, however, were evident in relation to many hematologic and biochemical parameters. Sub-adults had significantly greater values (mean \pm standard deviation) for $\mathrm{Hb}(16.0 \pm 2.9 \mathrm{~g} / \mathrm{dL})$, $\operatorname{TEC}\left(10.1 \pm 1.7 \times 10^{6} / \mu 1\right)$, and TLC $\left(19.1 \pm 4.1 \times 10^{6} / \mu 1\right)$ compared to adults (Hb: $14.4 \pm 2.1 \mathrm{~g} / \mathrm{dL}$; TEC: $8.4 \pm 1.9 \times$ 
Table-3: Comparison of hematology results for sub-adult, adult and geriatric Indian leopards (Panthera pardus fusca) from BBP and MLRC.

\begin{tabular}{|c|c|c|c|c|c|c|c|c|c|}
\hline \multirow[t]{2}{*}{ Parameters } & \multirow[t]{2}{*}{$\mathbf{n}$} & \multirow[t]{2}{*}{ Min } & \multirow[t]{2}{*}{ Max } & \multirow[t]{2}{*}{ Mean } & \multirow[t]{2}{*}{ SEM } & \multirow[t]{2}{*}{ SD } & \multicolumn{2}{|c|}{$\begin{array}{l}95 \% \text { CI of the } \\
\text { mean }\end{array}$} & \multirow[t]{2}{*}{ p value } \\
\hline & & & & & & & LCI & UCI & \\
\hline \multicolumn{10}{|l|}{ Sub-adults } \\
\hline $\operatorname{TLC}\left(10^{3} / \mu \mathrm{l}\right)$ & 19 & 10.3 & 26.4 & 19.1 & 0.9 & 4.1 & 17.2 & 20.9 & 0.027 \\
\hline $\operatorname{TEC}\left(10^{6} / \mu \mathrm{l}\right)$ & 19 & 6.8 & 12.7 & 10.1 & 0.4 & 1.7 & 9.4 & 10.8 & 0.001 \\
\hline $\mathrm{Hb}(\mathrm{g} / \mathrm{dl})$ & 19 & 10.5 & 20.2 & 16.0 & 0.7 & 2.9 & 14.7 & 17.2 & 0.05 \\
\hline PCV $(\%)$ & 19 & 32.8 & 65.4 & 49.9 & 1.9 & 8.4 & 46.1 & 53.6 & 0.537 \\
\hline $\mathrm{MCV}(\mathrm{fl})$ & 19 & 47.0 & 54.2 & 50.8 & 0.5 & 2.1 & 49.8 & 51.7 & 0.002 \\
\hline $\mathrm{MCH}(\mathrm{pg})$ & 19 & 14.2 & 18.4 & 16.3 & 0.2 & 1.1 & 15.8 & 16.8 & 0.009 \\
\hline $\mathrm{MCHC}(\%)$ & 19 & 29.7 & 33.6 & 31.9 & 0.1 & 1.2 & 33.7 & 34.0 & 0.112 \\
\hline \multicolumn{10}{|l|}{ Adults } \\
\hline $\operatorname{TLC}\left(10^{3} / \mu \mathrm{l}\right)$ & 38 & 8.7 & 27.9 & 15.8 & 1.1 & 6.8 & 13.6 & 17.9 & $-a$ \\
\hline $\operatorname{TEC}\left(10^{6} / \mu \mathrm{l}\right)$ & 38 & 5.4 & 11.8 & 8.4 & 0.3 & 1.9 & 7.8 & 9.0 & - \\
\hline $\mathrm{Hb}(\mathrm{g} / \mathrm{dl})$ & 38 & 11.0 & 20.2 & 14.4 & 0.4 & 2.1 & 13.7 & 15.1 & - \\
\hline PCV $(\%)$ & 38 & 34.0 & 60.9 & 48.5 & 1.0 & 6.4 & 46.4 & 50.5 & - \\
\hline $\operatorname{MCV}(\mathrm{fl})$ & 38 & 46.2 & 88.6 & 60.8 & 2.1 & 12.8 & 56.7 & 64.8 & - \\
\hline $\mathrm{MCH}(\mathrm{pg})$ & 38 & 13.6 & 23.9 & 18.8 & 0.6 & 3.4 & 17.8 & 19.9 & - \\
\hline $\mathrm{MCHC}(\%)$ & 38 & 25.6 & 34.2 & 30.4 & 0.6 & 3.4 & 29.4 & 31.5 & - \\
\hline \multicolumn{10}{|l|}{ Geriatric } \\
\hline $\operatorname{TLC}\left(10^{3} / \mu \mathrm{l}\right)$ & 26 & 8.7 & 27.9 & 14.7 & 1.1 & 5.8 & 12.5 & 16.9 & $-a$ \\
\hline TEC $\left(10^{6} / \mu \mathrm{l}\right)$ & 26 & 5.4 & 11.8 & 8.1 & 0.4 & 1.9 & 7.4 & 8.9 & - \\
\hline $\mathrm{Hb}(\mathrm{g} / \mathrm{dl})$ & 26 & 11.0 & 20.2 & 14.3 & 0.5 & 2.5 & 13.4 & 15.3 & - \\
\hline PCV $(\%)$ & 26 & 34.0 & 60.9 & 47.6 & 1.2 & 6.3 & 45.1 & 50.0 & - \\
\hline MCV (fl) & 26 & 46.2 & 88.55 & 61.3 & 2.1 & 10.7 & 57.1 & 65.4 & - \\
\hline $\mathrm{MCH}(\mathrm{pg})$ & 26 & 13.6 & 23.88 & 18.2 & 0.5 & 2.8 & 17.1 & 19.3 & - \\
\hline $\mathrm{MCHC}(\%)$ & 26 & 25.6 & 34.2 & 30.3 & 0.5 & 2.4 & 29.3 & 31.2 & - \\
\hline
\end{tabular}

${ }^{a} p$ values for sub-adult, adult and geriatric group comparison are listed in the top (sub-adult) half of the table. TLC=Total leukocyte count, $\mathrm{TEC}=$ Total erythrocyte count, $\mathrm{Hb}=$ Hemoglobin, $\mathrm{PCV}=$ Packed cell volume, $\mathrm{MCV}=\mathrm{Mean}$ corpuscular volume, $\mathrm{MCH}=$ Mean corpuscular hemoglobin, $\mathrm{MCHC}=$ Mean corpuscular hemoglobin concentration, $\mathrm{CI}=\mathrm{Confidence}$ interval, LCI=Lower confidence interval, UCI=Upper confidence interval, BBP=Bannerghatta Biological Park, MLRC=Manikdoh Leopard Rescue Center, SEM=Standard error of mean, SD=Standard deviation

$10^{6} / \mu 1$ and TLC: $\left.15.8 \pm 6.8 \times 10^{3} / \mu 1\right)$ and geriatric group (Hb: $14.3 \pm 2.5 \mathrm{~g} / \mathrm{dL}$; TEC: $8.1 \pm 1.9 \times 10^{6} / \mu 1$ and TLC: $\left.14.7 \pm 5.8 \times 10^{3} / \mu 1\right)$, whereas they had significantly lower MCV $(50.8 \pm 2.1 \mathrm{fl})$ and $\mathrm{MCH}(16.3 \pm 1.1 \mathrm{pg})$ compared to adults (MCV: $60.8 \pm 12.8 \mathrm{fl}$ and $\mathrm{MCH}$ : $18.8 \pm 3.4 \mathrm{pg}$ ) and geriatric group (MCV: $61.3 \pm 10.7$ fl and MCH: $18.2 \pm 2.8 \mathrm{pg}$ ). Among, serum biochemistry parameters the sub-adult age group were observed to have significantly lower values for TP $(6.5 \pm 1.4 \mathrm{~g} / \mathrm{dl})$ and ALT $(25.8 \pm 14.5 \mathrm{IU} / \mathrm{L})$ than adults (TP: $7.0 \pm 1.4 \mathrm{~g} / \mathrm{dl}$ and ALT: $46.8 \pm 26.8 \mathrm{IU} / \mathrm{L}$ ) and geriatric leopards (TP: $7.3 \pm 1.4 \mathrm{~g} / \mathrm{dl}$ and $53.1 \pm 32.1 \mathrm{IU} / \mathrm{L}$ ) age groups.

\section{Discussion}

Flagging hematology and biochemistry values obtained from an animal as either normal or abnormal is the first interpretive step in interpreting hemato-biochemical reports. However, this is not as simple as it may seem in wild animals as reference intervals are usually based on limited population testing and do not account for variation within subpopulations defined by age, sex, subspecies, or extrinsic factors such as instrumentation and phlebotomy techniques. Further, adequate numbers of normal animals must be sampled to arrive at intervals that are valid for healthy animals from the defined population. Thus, unlike published studies [16], sampling large numbers of animals to make the results most reflective of the healthy population is highly desirable.

A reference interval is typically defined as values encompassing the median $95 \%$ of a tested population of apparently healthy animals [11]. Inherent in this definition is that $2.5 \%$ of the healthy population will have values outside either side of the median $95 \%$, suggesting they are abnormal [11]. Thus, the nonparametric method used to arrive at reference intervals in this study, wherein the test values were rank ordered, and any outliers were removed and then the middle $95 \%$ of test results define the reference interval are less biased, compared to other methods $[9,11,20]$.

In felids, the number, size and $\mathrm{Hb}$ concentration of circulating erythrocytes is known to increase gradually after 1 month of age and, at 3-4 months reaches values similar to those of adults depending on the amount of iron in the diet [21]. However, this study showed significantly greater TEC and $\mathrm{Hb}$ counts in sub-adults compared to adult and geriatric animals. This might be subsequent to transient polycythemia from splenic contraction [22], a momentary response to epinephrine due to stress, anger, and fear as observed in sub-adults, most of them with a history of being and the $\mathrm{RBC}$ counts are known to revert to normal in a short period [22].

Higher TLC observed in sub-adults may also be indicative of low-level stressor factors inherent with 
Table-4: Comparison of serum biochemistry results for sub-adult, adult and geriatric Indian leopards (Panthera pardus fusca) from BBP and MLRC.

\begin{tabular}{|c|c|c|c|c|c|c|c|c|c|}
\hline \multirow[t]{2}{*}{ Parameter } & \multirow[t]{2}{*}{$\mathbf{n}$} & \multirow[t]{2}{*}{ Minimum } & \multirow[t]{2}{*}{ Maximum } & \multirow[t]{2}{*}{ Mean } & \multirow[t]{2}{*}{ SEM } & \multirow[t]{2}{*}{ SD } & \multicolumn{2}{|c|}{$\begin{array}{c}95 \% \text { CI of the } \\
\text { mean }\end{array}$} & \multirow[t]{2}{*}{ p value } \\
\hline & & & & & & & LCI & UCI & \\
\hline \multicolumn{10}{|l|}{ Sub adult } \\
\hline TP (g/dl) & 19 & 4.3 & 11.1 & 6.5 & 0.3 & 1.4 & 5.9 & 7.2 & 0.01 \\
\hline Albumin $(\mathrm{g} / \mathrm{dl})$ & 19 & 1.9 & 5.0 & 4.0 & 0.2 & 0.8 & 3.6 & 4.3 & 0.91 \\
\hline Globulin ( $\mathrm{g} / \mathrm{dl})$ & 19 & 0.3 & 6.6 & 2.7 & 0.3 & 1.4 & 2.0 & 3.3 & 0.168 \\
\hline AST (IU/L) & 19 & 9.9 & 77.7 & 39.6 & 4.5 & 19.8 & 30.7 & 48.5 & 0.561 \\
\hline ALT (IU/L) & 19 & 2.0 & 50.2 & 25.8 & 3.3 & 14.5 & 19.2 & 32.3 & 0.001 \\
\hline Creatinine (mg/dl) & 19 & 0.4 & 4.2 & 1.7 & 0.2 & 1.0 & 1.2 & 2.1 & 0.213 \\
\hline BUN (mg/dl) & 19 & 7.5 & 56.1 & 25.7 & 2.7 & 11.9 & 20.4 & 31.1 & 0.645 \\
\hline Glucose $(\mathrm{mg} / \mathrm{dl})$ & 19 & 14.1 & 159.0 & 66.1 & 7.3 & 31.8 & 51.8 & 80.3 & 0.249 \\
\hline Triglycerides (mg/dl) & 19 & 4.0 & 52.9 & 27.4 & 3.0 & 13.0 & 21.5 & 33.2 & 0.62 \\
\hline $\mathrm{Ca}(\mathrm{mg} / \mathrm{dl})$ & 19 & 3.5 & 27.0 & 9.3 & 1.1 & 5.0 & 7.1 & 11.6 & 0.193 \\
\hline$P(\mathrm{mg} / \mathrm{dl})$ & 19 & 2.6 & 12.4 & 6.2 & 0.5 & 2.1 & 5.2 & 7.2 & 0.181 \\
\hline \multicolumn{10}{|l|}{ Adult } \\
\hline $\mathrm{TP}(\mathrm{g} / \mathrm{dl})$ & 38 & 4.9 & 10.1 & 7.0 & 0.2 & 1.4 & 6.6 & 7.4 & $-a$ \\
\hline Albumin $(\mathrm{g} / \mathrm{dl})$ & 38 & 1.8 & 6.7 & 3.9 & 0.2 & 1.2 & 3.6 & 4.3 & - \\
\hline Globulin ( $\mathrm{g} / \mathrm{dl})$ & 38 & 0.3 & 5.2 & 3.0 & 0.2 & 1.2 & 2.6 & 3.4 & - \\
\hline AST (IU/L) & 16 & 13.7 & 49.0 & 45.3 & 5.2 & 20.7 & 35.1 & 55.4 & - \\
\hline ALT (IU/L) & 38 & 21.3 & 147.0 & 46.8 & 4.4 & 26.8 & 38.3 & 55.3 & - \\
\hline Creatinine $(\mathrm{mg} / \mathrm{dl})$ & 38 & 0.5 & 2.5 & 1.7 & 0.1 & 0.6 & 1.5 & 1.8 & - \\
\hline BUN (mg/dl) & 38 & 3.1 & 56.3 & 27.5 & 1.8 & 11.4 & 23.9 & 31.1 & - \\
\hline Glucose (mg/dl) & 16 & 24.1 & 66.2 & 53.5 & 5.6 & 22.5 & 42.4 & 64.5 & - \\
\hline Triglycerides (mg/dl) & 16 & 22.9 & 43.9 & 31.1 & 3.4 & 13.6 & 24.4 & 37.8 & - \\
\hline $\mathrm{Ca}(\mathrm{mg} / \mathrm{dl})$ & 38 & 7.3 & 14.2 & 10.0 & 0.4 & 2.5 & 9.2 & 10.8 & - \\
\hline$P(\mathrm{mg} / \mathrm{dl})$ & 38 & 2.22 & 12.4 & 5.4 & 0.3 & 1.6 & 4.9 & 5.9 & - \\
\hline \multicolumn{10}{|l|}{ Geriatric } \\
\hline TP (g/dl) & 26 & 4.9 & 10.08 & 7.3 & 0.2 & 1.2 & 6.9 & 7.8 & $-a$ \\
\hline Albumin $(\mathrm{g} / \mathrm{dl})$ & 26 & 1.8 & 6.7 & 3.8 & 0.2 & 1.0 & 3.5 & 4.2 & - \\
\hline Glo (g/dl) & 26 & 0.3 & 5.23 & 3.4 & 0.2 & 1.1 & 2.9 & 3.8 & - \\
\hline AST (IU/L) & 6 & 13.7 & 49 & 36.5 & 5.5 & 13.6 & 25.7 & 47.4 & - \\
\hline ALT (IU/L) & 26 & 21.3 & 146.7 & 53.1 & 6.3 & 32.1 & 40.8 & 65.5 & - \\
\hline Creatinine (mg/dl) & 26 & 0.5 & 2.54 & 1.4 & 0.1 & 0.5 & 1.2 & 1.6 & - \\
\hline BUN (mg/dl) & 26 & 3.1 & 56.3 & 29.0 & 2.2 & 11.3 & 24.6 & 33.3 & - \\
\hline Glucose $(\mathrm{mg} / \mathrm{dl})$ & 6 & 24.1 & 66.2 & 48.9 & 6.0 & 14.8 & 37.1 & 60.8 & - \\
\hline Triglycerides (mg/dl) & 6 & 22.9 & 43.9 & 31.7 & 3.0 & 7.3 & 25.8 & 37.5 & - \\
\hline $\mathrm{Ca}(\mathrm{mg} / \mathrm{dl})$ & 26 & 7.3 & 14.21 & 10.1 & 0.4 & 2.2 & 9.3 & 11.0 & - \\
\hline$P(\mathrm{mg} / \mathrm{dl})$ & 26 & 2.2 & 12.4 & 5.2 & 0.4 & 1.9 & 4.5 & 5.9 & - \\
\hline
\end{tabular}

ap values for sub-adult, adult and geriatric group comparison are listed in the top (sub-adult) half of the table. TP=Total protein, $\mathrm{AST}=$ Aspartate aminotransferase, $\mathrm{ALT}=$ Alanine aminotransferase, $\mathrm{BUN}=$ Blood urea nitrogen, $\mathrm{Ca}=\mathrm{Serum}$ calcium, $\mathrm{P}=$ Serum phosphorus, $\mathrm{CI}=$ Confidence interval, $\mathrm{LCI}=$ Lower confidence interval, UCI=Upper confidence interval, $\mathrm{BBP}=$ Bannerghatta Biological Park, $\mathrm{MLRC}=$ Manikdoh Leopard Rescue Center, $\mathrm{SEM}=$ Standard error of mean, $\mathrm{SD}=\mathrm{Standard}$ deviation

the animals newly introduced to captive environment. The significantly lesser MCV and MCH values observed in sub-adults, in comparison to geriatric and adult age groups leopards can be attributed to the age and dietary differences that permit older age classes to produce relatively larger erythrocytes than younger age classes; similar to the patterns observed in other carnivores $[20,23,24]$.

Among serum biochemistry parameters, subadults had significantly lower TP compared to adults and geriatric leopards. The increased serum concentrations of TPs in the older age groups could be explained as an age-related phenomenon, having been observed in other carnivores $[25,26]$ and also probably due, in part, to the increase in gammaglobulins induced by either vaccinations or increased contact with environmental microorganisms [27]. Further, the TP levels for immature and growing animals tend to be normally low, consistent with the expected higher physiological demand for proteins during their growth phase [28].

ALT and AST are used as general indicators of liver function, with ALT being more liver-specific [29]. In this study, older age classes were observed to have higher ALT activity compared to young animals and in particular, sub-adults had significantly lower ALT levels than geriatric animals. This observation in older age classes can be attributed to physiological variations related with age, hormone action, reproductive phases as well as exposure of liver to a wide array of toxins, infectious, agents, drug metabolites and endotoxins over time [30].

\section{Conclusion}

This study provides a comprehensive analysis of hematologic and biochemical parameters for 
Indian leopards, including the possible variations in these parameters based on age and sex. To the best of authors' knowledge, there are no similar published reference intervals available for the subspecies and thus the baselines provided here will permit better captive management of the subspecies, serve as a guide to assess the health and physiological status of the free ranging leopards, and may contribute valuable information for making effective management decisions during translocation or rehabilitation process.

\section{Authors' Contributions}

AAS and SKM conceived and designed the study. AAS, SKM, AD, SS and PK were involved in collection and compilation of data from BBP and MLRC. MV and LJ performed the hematology serum biochemistry analysis. AN carried out all the statistical analysis required for the manuscript. AAS, SKM, $\mathrm{AD}, \mathrm{SS}, \mathrm{PK}, \mathrm{MV}, \mathrm{AN}$, and $\mathrm{LJ}$ contributed to the manuscript writing and the reviewing of the literature. All authors read and approved the final manuscript.

\section{Acknowledgments}

Authors did not receive any external funding for the study. The authors are thankful to Mr. Range Gowda, Executive Director, Bannerghatta Biological Park and Mr. Kartick Satyanarayan, Co-Founder and Chairmen, Wildlife SOS, for providing access to the data and extending their logistical and laboratory support during the study. The authors would also like to acknowledge the Department of Biotechnology, Jain University, Bengaluru, for their technical support. We also thank the staff at Bannerghatta Zoo, Bannerghatta - Central Zoo Authority Rescue Center, and Manikdoh Leopard Rescue Center for their valuable field assistance during the study.

\section{Competing Interests} interest.

The authors declare that they have no competing

\section{References}

1. Gubbi, S., Poornesha, H.C., Daithota, A. and Nagashettihalli, H. (2014) Roads emerging as a critical threat to leopards in India. Cat News, 60: 30-31.

2. Stein, A.B., Athreya, V., Gerngross, P., Balme, G., Henschel, P., Karanth, U., Miquelle, D., Rostro, S., Kamler, J.F. and Laguardia, A. (2016). In: Panthera pardus. The IUCN Red List of Threatened Species; 2016. http://www.dx.doi.org/10.2305/IUCN.UK.2016-1.RLTS. T15954A50659089. Accessed on 10-10-2016.

3. Prater, S.H. (1980) In: The Book of Indian Animals. Bombay Natural History Society and Oxford University Press, Bombay. p324.

4. Chauhan, D.S. and Goyal, S.P. (2000) A Study on Distribution, Relative Abundance and Food Habits of Leopard (Panthera pardus) in Garhwal Himalayas. Technical Report, Wildlife Institute of India. Available from: http://www.carnivorecology.free.fr/pdf/leopardgarhwal.PDF. Accessed on 20-10-2016.

5. Central Zoo Authority. (2016) Leopard. In: Inventory Report. Available from: http://www.cza.nic.in/inventory. html. Accessed on 20-10-2016.
6. Franson, J.C., Hoffman, D.J. and Schmutz, J.A. (2009) Plasma biochemistry values in emperor geese (Chen canagica) in Alaska: Comparisons among age, sex, incubation, and molt. J. Zoo Wildl. Med., 40(2): 321-327.

7. Brock, P., Hall, A., Goodman, S., Cruz, M. and AcevedoWhitehouse, K. (2013) Applying the tools of ecological immunology to conservation: A test case in the Galapagos sea lion. Anim. Conserv., 16: 19-31.

8. López-Ba, J.V., Sazatornil, V., Llaneza, L. and Rodríguez, A. (2013) Indirect effects on health and conservation and wolf persistence of contradictory policies that threaten traditional free-ranging horse husbandry. Conserv. Lett., 6: 448-455.

9. Santos, N., Maior, H.R., Nakamura, M., Roque, S., Brandão, R., Petrucci-Fonseca, F., Palacios, V., Garcia, E., López-Bao, J.V., Llaneza, L. and Álvares, F. (2015) Hematology and serum biochemistry values of free-ranging Iberian wolves (Canis lupus) trapped by leg-hold snares. Eur. J. Wildl. Res., 61(1): 135-141.

10. DelGiudice, G., Mech, D. and Seal, U. (1991) Gray wolf density and its association with weights and hematology of pups from 1970 to 1988. J. Wildl. Dis., 27(4): 630-636.

11. Weiser, G. and Allison, R.W. (2012) Perspectives in laboratory data interpretation and disease diagnosis. In: Thrall, M.A., Weiser, G., Allison, R.W. and Campbell, T.W. editors. Veterinary Hematology and Clinical Chemistry. $2^{\text {nd }}$ ed. John Wiley \& Sons, New Jersey. p40-50.

12. Caro, T.M., Holt, M.E., FitzGibbon, C.D., Bush, M., Hawkey, C.M. and Kock, R.A. (1987) Health of adult free-living cheetahs. J. Zool., 212(4): 573-584.

13. Seal, U.S., Plotka, E.D. and Gray, C.W. (1978) Baseline hematology, serum chemistry, and hormone data for captive tigers (Panthera tigris spp) and lions ( $P$. leo). In: International Tiger Studbook: Congress Report on $1^{\text {st }}$ International Symposium on the Management and Breeding of the Tiger. p174-192.

14. Dunbar, M.R., Nol, P. and Linda, S.B. (1997) Hematologic and serum biochemical reference intervals for Florida panthers. J. Wildl. Dis., 33(4): 783-789.

15. Maas, M., Keet, D.F. and Nielen, M. (2013) Hematologic and serum chemistry reference intervals for free-ranging lions (Panthera leo). Res. Vet. Sci., 95(1): 266-268.

16. Sabapara, R.H., Jani, R.G. and Bhuva, C.N. (2008) Haematological reference intervals for Indian leopards (Panthera pardus). Vet. World, 1(6): 173-174.

17. Teare, J.A. (2013) Panthera pardus. A CD-ROM resource. In: ISIS Physiological Reference Intervals for Captive Wildlife. International Species Information System, Eagan, MN. Available from: http://www.Panthera_pardus No Selection by gender All Ages Combined Standard_International_Units_2013_CD.html. Accessed on 12-01-2016.

18. Tryland, M. (2006) "Normal" serum chemistry values in wild animals. Vet. Rec., 158: 211-212.

19. Snedecor, G.W. and Cochran, W.B. (1996) In: Statistical Methods. $6^{\text {th }}$ ed. Oxford and IBH Publishing, New Delhi. p138-169.

20. Kusak, J., Rafaj, R.B., Zvorc, Z., Huber, D., Foršek, J., Bedrica, L. and Mrljak, V. (2005) Effects of sex, age, body mass and capturing method on hematologic values of brown bears in Croatia. J. Wildl. Dis., 41(4): 843-847.

21. Anderson, L., Wilson, R. and Hay, D. (1971) Haematological values in normal cats from four weeks to one year of age. Res. Vet. Sci., 12(6): 579-583.

22. Thrall, M.A. (2012) Classification of and diagnostic approach to polycythemia. In. Thrall, M.A., Weiser, G., Allison, R.W. and Campbell, T.W. editors. Veterinary Hematology and Clinical Chemistry. $2^{\text {nd }}$ ed. John Wiley \& Sons, New Jersey. p121-124.

23. Seal, U.S., Swaim, W.R. and Erickson, A.W. (1967) Hematology of the Ursidae. Comp. Biochem. Physiol., 22: 451-460.

24. Castellanos, A., Arias, L., Jackson, D. and Castellanos, R. 
(2010) Hematological and serum biochemical values of Andean bears in Ecuador. Ursus, 21(1): 115-120.

25. Lowseter, L.A., Gillet, N.A., Gerlach, R.F.B. and Muggenvurg, B.A. (1990) The effects of aging on hematology and serum chemistry values in the beagle dog. Vet. Clin. Pathol., 19: 13-19.

26. Padmanath, K., Dash, D., Behera, P.C., Sahoo, N., Sahoo, G., Subramanian, S. and Bisoi, P.C. (2015) Biochemical reference values of captive Royal Bengal tigers (Panthera tigris tigris) in Orissa, India. Int. J. Adv. Res. Biol. Sci., 2(4): 274-278.

27. Mundim, A.V., Coelho, A.O., Hortêncio, S.M., Guimarães, E.C. and Espindola, F.S. (2007) Influence of age and sex on the serum biochemical profile of Doberman dogs in the growth phase. Comp. Clin. Pathol., 16(1): 41-46.
28. McMichael, L., Edson, D., McLaughlin, A., Mayer, D., Kopp, S., Meers, J. and Field, H. (2015) Haematology and plasma biochemistry of wild black flying-foxes, (Pteropus alecto) in Queensland, Australia. PloS One, 10(5): e0125741.

29. Wu, D., Yi, Y., Sun, F., Zhou, L., Yang, F., Wang, H., Zhang, G., Zhang, Y.A. and Yue, F. (2014) Effects of age and sex on the hematology and blood chemistry of Tibetan macaques (Macaca thibetana). J. Am. Assoc. Lab. Anim. Sci., 53(1): 12-17.

30. Harper, J.E., Hackett, R.M., Wilkinson, J. and Heaton, P.R. (2003) Age-related variations in hematologic and plasma biochemical test results in beagles and labrador retrievers. J. Am. Vet. Med. Assoc., 223: 14.

$* * * * * * * *$ 